\title{
D-Glucuronyl C5-epimerase suppresses small-cell lung cancer cell proliferation in vitro and tumour growth in vivo
}

\author{
EV Grigorieva*,I,2, TY Prudnikova', NV Domanitskaya', LA Mostovich', TV Pavlova ${ }^{2}$, VI Kashuba ${ }^{2,3}$ and \\ ER Zabarovsky ${ }^{2,4}$
}

'Institute of Molecular Biology and Biophysics SD RAMS, Timakova str 2, Novosibirsk 630 I 17, Russia; ${ }^{2}$ MTC, Sodersjukhuset, Karolinska Institute, Stockholm, Sweden; ${ }^{3}$ Institute of Molecular Biology and Genetics, Ukrainian National Academy of Sciences, Kiev, Ukraine; ${ }^{4}$ Engelhard Institute of Molecular Biology RAS, Moscow, Russia

\begin{abstract}
BACKGROUND: D-Glucuronyl C5-epimerase (GLCE) is a key enzyme involved in the biosynthesis of heparan sulphate proteoglycans, which has an important role in cell-cell and cell-matrix interactions and signalling. Decreased GLCE expression in human breast tumours and its anti-proliferative effects in breast cancer cells suggest that it may be a candidate tumour-suppressor gene. The aim of this study was to investigate the involvement of GLCE in lung carcinogenesis.

METHODS: D-Glucuronyl C5-epimerase expression in different lung cancer cell lines was determined and the gene was ectopically re-expressed in U2020 small-cell lung cancer cells. Cellular proliferation in vitro and tumour growth in vivo were then examined. RESULTS: Ectopic re-expression of GLCE in U2020 cells did not affect cell viability but did influence morphology. Cellular proliferation in vitro and tumour formation in vivo were both suppressed. These effects were mediated via downregulation of several pro-angiogenic growth factors and their receptors, including VEGF-A, TGFBI, FGFR2, PDGF-A and PDGF-B, and TNFa and its receptors. Expression of matrix metalloproteinase2, MTAI, PLAU, TIMP3, SI O0A4, SERPINEI and TWISTI was also downregulated. CONCLUSION: The anti-tumour effects associated with ectopic GLCE re-expression suggest that it may be a potential tumour-suppressor gene and a possible target for lung cancer diagnosis and treatment.
\end{abstract}

British Journal of Cancer (20II) I 05, 74-82. doi:I0.1038/bjc.20 I I.I70 www.bjcancer.com

Published online 7 June 201I

(c) 20II Cancer Research UK

Keywords: heparan sulphate proteoglycan; D-Glucuronyl C5-epimerase; small-cell lung cancer; tumour-suppressor gene; angiogenesis; metastasis

Lung cancer is one of the most common cancers worldwide in terms of both incidence and mortality, with 1.35 million new cases and 1.18 million deaths per year. Numerous attempts have been made to find reliable molecular markers and possible new targets to treat the disease (Hann and Rudin, 2007); however, despite extensive studies, the molecular mechanisms underlying lung carcinogenesis remain unclear (Rosti et al, 2006; Pastorino, 2010).

The aim of the present study was to examine the possible involvement of D-glucuronyl C5-epimerase (GLCE) in the development of lung cancer. D-Glucuronyl C5-epimerase is one of the key enzymes involved in the biosynthesis of heparan sulphate (HS): the polysaccharide moiety of complex HS proteoglycan (HSPG) molecules. Heparan sulphate proteoglycans are widely expressed on cell surfaces and within the extracellular matrix (ECM). They have important roles in cell-cell and cell-matrix interactions and signalling through their interactions with various proteins, including growth factors and their receptors (Ori et al, 2008). The most common HSPGs are syndecans (Alexopoulou et al, 2007), glypicans (Filmus et al, 2008), perlecan (Whitelock et al, 2008), collagen XVIII and agrin (Iozzo, 2005). Abnormal expression or dysregulated function of HSPGs is involved in cancer and

*Correspondence: Dr EV Grigorieva; E-mail: elv_grig@yahoo.com Revised II April 20I I; accepted I5 April 20 II; published online 7 June 2011 angiogenesis, and is crucial for the evolution of the tumour microenvironment (Iozzo et al, 2009).

At present, the functional contribution of glycosylation (particularly proteoglycans) to lung carcinogenesis is unclear; although the pattern of HSPG expression is significantly altered in lung tumours and may contribute to their invasive phenotype (Nackaerts et al, 1997), and the expression of the candidate tumour-suppressor gene, glypican-3, is absent in all the non-smallcell lung carcinoma cell lines tested to date (Kim et al, 2003). However, because there are few reliable and sensitive methods available for investigating the role of glycosylation, the majority of studies have only looked at the HSPG protein core. Nevertheless, in many cases, glycosylation is a major determinant of HSPG function (Blackhall et al, 2001; Reijmers et al, 2010). An alternative approach to the problem could be to study the biosynthetic machinery for HS, as defects in biosynthesis would, presumably, affect all HSPGs (Lander and Selleck, 2000).

Little is known about the functional role of HS biosynthetic enzymes in carcinogenesis, and GLCE is the least studied of all the enzymes (Nadanaka and Kitagawa, 2008). D-Glucuronyl C5-epimerase is responsible for the epimerisation of D-glucuronic acid (D-GlcUA) to form L-iduronic acid (L-IdoUA), which provides flexibility to the HS chains and is important for their interactions with various growth factors (Catlow et al, 2008; Jia et al, 2009).

D-Glucuronyl C5-epimerase has been cloned from bovine lung cDNA (Li et al, 1997), murine liver (Li et al, 2001) and mouse 
mastocytoma cell line cDNA libraries (Crawford et al, 2001). Murine (NM_033320), bovine (NM_174070) and human (NM_015554) epimerase cDNAs are $82-86 \%$ homologous at the nucleotide level and 96-99\% homologous at the amino-acid level. Chromosome mapping suggests the occurrence of a single epimerase gene on chromosome 15q23 (Li et al, 2001). The involvement of GLCE in the aetiology and pathology of human disease is not known.

Recent studies show decreased expression of GLCE in human breast tumours (Eshchenko et al, 2007; Grigorieva et al, 2008). Ectopic expression of GLCE inhibits the proliferation of MCF7 cells in vitro through attenuated expression of the different genes involved in cell cycle regulation, angiogenesis and metastasis (Prudnikova et al, 2010). These data support the involvement of the gene in breast carcinogenesis.

According to the data presented at http://www.genecards.org/ cgi-bin/carddisp.pl?gene $=$ GLCE $\&$ search $=$ GLCE, another tissue showing relatively high GLCE expression is human lung. Targeted disruption of the GLCE gene in mice results in structurally altered HS, which lacks IdoA and carries a lethal phenotype resulting in lung defects, renal agenesis and skeletal malformations (Li et al, 2003). These data suggest that appropriate GLCE expression/ activity is particularly important in lung tissues; thus, we hypothesised that dysregulation of GLCE expression in human lung tissue may be a significant factor in the aetiology and/or development of malignancy.

In this study, we investigated the expression levels of GLCE in different lung cancer cell lines and ectopically expressed GLCE in the small-cell lung cancer cell line, U2020. The proliferation rates of the cells in vitro and tumour growth in vivo were studied, as well as the possible molecular mechanisms of the functional effect of GLCE in lung cancer.

\section{MATERIALS AND METHODS}

\section{Cell lines and cell culture}

The U2020, A549, H157 and H647 human lung cancer cell lines were obtained from MTC (Karolinska Institute, Stockholm, Sweden). The A716 cell line was a kind gift from Professor M Lerman (Center for Cancer Research, National Cancer Institute, Frederick, MD, USA). All cell lines were maintained in Iscove's Modified Dulbecco's medium (IMDM) supplemented with

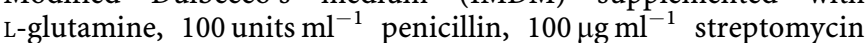
and $10 \% \mathrm{FBS}$ at $37^{\circ} \mathrm{C}$ in a humidified $5 \% \mathrm{CO}_{2}$ incubator. Cells were harvested for passaging or analysis using trypsin/EDTA.

\section{Analyses of GLCE expression using RT - PCR}

Total RNA was extracted from the cells using the PureLink Total RNA Purification System (Invitrogen, Carlsbad, CA, USA) according to the manufacturer's instructions. cDNA was synthesised from $1-2 \mu \mathrm{g}$ of total RNA using a First Strand cDNA Synthesis kit (Fermentas, Hanover, MD, USA) and 1/10th of the product was subjected to PCR analysis.

The following conditions were used for multiplex RT-PCR: $95^{\circ} \mathrm{C}$ for $10 \mathrm{~min}, 95^{\circ} \mathrm{C}$ for $15 \mathrm{~s}, 59^{\circ} \mathrm{C}$ for $15 \mathrm{~s}$ and $72{ }^{\circ} \mathrm{C}$ for $1 \mathrm{~min}$, with a final elongation step at $72{ }^{\circ} \mathrm{C}$ for $10 \mathrm{~min}$ using a Tercik PCR machine (DNA-technology, Moscow, Russia). The total reaction volume was $20 \mu$ l. D-Glucuronyl C5-epimerase and GAPDH (housekeeping gene) were amplified for 32 and 20 cycles, respectively. The amplified products were separated on $1.2 \%$ agarose gels. The gels were scanned using the 'DNA Analyzer' system (Vilber Lourmat, Marne-la-Valle, France) and epimerase expression levels were estimated from the intensity of the amplified GLCE DNA fragment normalised against the intensity of GAPDH (TotalLab programme, Nonlinear Dynamics, Newcastle upon Tyne, UK). The PCR primers used for human GLCE and GAPDH were as follows:
GLCE-F, 5'-AAGGGAGACGAGAGGGGAACGAA-3'; GLCE-R, 5'-G CCACCTTTCTCATCCTGGTTC-3'; GAPDH-F, $5^{\prime}$-GGGCGCCTGGT CACAA-3'; GAPDH-R, 5'-AACATGGGGGCATCAGCAGA-3'. Quantitative real-time RT - PCR ( $\mathrm{qRT}$ - PCR) was performed using the BioRad IQ5 Multicolor Real-Time PCR Detection System (BioRad, Hercules, CA, USA) and the GLCE TaqMan Custom Assay (Applied Biosystems, Foster City, CA, USA) under the following conditions: $95^{\circ} \mathrm{C}$ for $3 \mathrm{~min}$, followed by 40 cycles at $95^{\circ} \mathrm{C}$ for $10 \mathrm{~s}$ and $60{ }^{\circ} \mathrm{C}$ for $30 \mathrm{~s}$. The total reaction volume was $25 \mu \mathrm{l}$. $\beta$-actin $(A C T B)$ was used as the housekeeping gene. The PCR primers and TaqMan probes used were: GLCE-F, $5^{\prime}$-TTCCAAAGTCTATGCAC AGAGAGC-3'; GLCE-R, 5' -TCCACATTGTAGCCTTCAAAAGACA$3^{\prime}$; GLCE probe, 5'-FAM-CCCCTATCACCCCGATGGT-TAMRA-3'; $\beta$-actin-F, $5^{\prime}$-GGCACCCAGCACAATGAAG- $3^{\prime} ; \beta$-actin-R, $5^{\prime}$-GCCG ATCCACACGGAGTACT- $3^{\prime} ; \quad \beta$-actin-probe, $5^{\prime}$-FAM-TCAAGATC ATTGCTCCTCCTGAGCGC-TAMRA- ${ }^{\prime}$.

\section{Construction of the epimerase-expression plasmid}

Human GLCE (NM_015554) was cloned into the episomal vector, pETE/Bsd (Protopopov et al, 2002). The full-length GLCE cDNA sequence was amplified from the KIAA0836 clone (AB_020643) encoding the $5^{\prime}$-truncated sequence of GLCE (Kazusa DNA Research Institute, Chiba, Japan) by PCR using Pfu-DNApolymerase (Stratagene, La Jolla, CA, USA) and the epi-oligoF 5'-CTAAGATCTAGATATGCGTTGCTTGGCAGCTCGGGTCAACT ATAAGACTTTGATTATTA- $3^{\prime}$ and epi-R 5'-TACAGCGGCCGCT GAAGTGCAGTTTTGGT- $3^{\prime}$ primers. The amplified full-length DNA fragment was then cloned into the pETE/Bsd vector at the BglII and NotI sites. The GLCE sequence was verified by sequencing (Dye Terminator Cycle sequencing Kit and an ABI PRISM 3700 Genetic Analyzer, Applied Biosystems, Foster City, CA, USA).

\section{Transfection and selection of stably transfected epi-U2020 cell clones}

To obtain stable cell clones expressing GLCE, U2020 cells were transfected with epi-pETE/Bsd or pETE/Bsd plasmid DNA $(0.5 \mu \mathrm{g}$ DNA per well) in 12-well plates using Lipofectamine and Plus Reagent (Invitrogen) according to the manufacturer's protocol. Transiently transfected epi-U2020 and pETE-U2020 cells were cultured for 2-3 weeks in IMDM medium containing Bsd $\left(5 \mu \mathrm{g} \mathrm{ml}^{-1}\right)$ to select stable clones.

\section{Immunocytochemistry}

For immunofluorescence, cells were grown on glass coverslips and then fixed with $4 \%$ formaldehyde. The anti-GLCE custom rabbit polyclonal serum (GenScript Corporation, Piscataway, NJ, USA) was used for immunostaining. Staining patterns were visualised with TexasRed-conjugated antibody against rabbit IgGs (Vector Laboratories, Burlingame, CA, USA) (1:1000 dilution). The nuclei were mounted and counterstained with DAPI using SlowFade Gold with DAPI mounting medium (Invitrogen) and observed by fluorescent microscopy (Axio Imager, Carl Zeiss UK, Hertfordshire, UK).

\section{Colony formation assay}

Transiently transfected cells were stripped $24-48 \mathrm{~h}$ after transfection and plated on $100 \mathrm{~mm}^{2}$ cell culture dishes at a density of 500-1000 cells per plate. After selection with Bsd $\left(5 \mu \mathrm{g} \mathrm{ml}^{-1}\right)$, Giemsa-stained colonies were photographed and counted using Quantity One software (version 4.4.0; Bio-Rad, Hercules, CA, USA).

\section{Growth curve assay}

The growth curves for the U2020 cells transiently transfected with epi-pETE/Bsd or pETE/Bsd plasmid DNA were monitored by 
direct cell counting. The cells were seeded at $10^{4}$ cells per well in 6-well plates $24 \mathrm{~h}$ after transfection, detached using $0.25 \%$ trypsin every $24 \mathrm{~h}$ and counted using a Coulter Counter (Beckman Coulter, Brea, CA, USA).

\section{In vitro cell proliferation assay}

Cell proliferation rate was determined using the CyQUANT NF Cell Proliferation Assay (Invitrogen) according to the manufacturer's protocol. Briefly, cells were plated in a 96-well microplate at densities of $100-500$ per well (8-12 identical wells in total) and the DNA content of the wells was measured every $24 \mathrm{~h}$. This was achieved by removing the medium and adding $50 \mu \mathrm{l}$ of fluorescent dye followed by incubation for $30 \mathrm{~min}$ at $37^{\circ} \mathrm{C}$. The fluorescence intensity of each sample was measured at $485 / 530 \mathrm{~nm}$ using fluorescence microplate reader (SPECTRA max, Molecular Devices, Sunnyvale, CA, USA).

\section{Tumour growth in SCID mice}

The tumourigenicity of epimerase-transfected U2020 and control pETE-U2020 cells was assessed by subcutaneously injecting the cells into 6-8-week-old SCID mice as previously described (Protopopov et al, 2002). Cell inoculation, monitoring of tumour growth and animal care were undertaken at the Karolinska Institute Animal House according to the Institutional guidelines. Briefly, cells were collected by centrifugation at 800 r.p.m. for 2 min and resuspended in serum-free IMDM medium at a concentration $2-3 \times 10^{6}$ cells per $100 \mu \mathrm{l}$ injection. The cells were inoculated together with a Matrigel matrix (BD Biosciences, Erembodegem, Belgium) according to the manufacturer's protocol. Mice were observed for tumour formation twice per week and the tumour size was measured using callipers.

\section{Cancer PathFinder RT2 Profiler PCR array}

The Cancer PathFinder RT2 Profiler PCR array (SABioscience, Frederick, MD, USA) was used to identify the molecular mechanisms underlying the anti-proliferative effects of GLCE. Briefly, total RNA was isolated using a RNAqueous Micro Kit (Applied Biosystems). The RNA concentration was determined using a Quant-iT Assay Kit for RNA quantification (Invitrogen) and was verified by electrophoresis. cDNA was synthesised from $1-2 \mu \mathrm{g}$ of total RNA using a First Strand cDNA Synthesis kit (Fermentas). Real-Time PCR was performed using an RT2 Profiler PCR Array System with SYBR Green PCR Master Mix (Fermentas) and an iCycler iQ5 Multicolor Detection System (Bio-Rad) according to the manufacturer's instructions. All data were analysed using Excel-based PCR Array Data Analysis Software (SABioscience). This integrated web-based software package automatically calculates ddCt-based fold changes in genes expression from the uploaded raw threshold cycle data. Each replicate cycle threshold $(\mathrm{Ct})$ was normalised to the average $\mathrm{Ct}$ of five endogenous controls (B2M, HPRT1, RPL13A, GAPDH and ACTB) on a per plate basis.

\section{RESULTS}

\section{D-Glucuronyl C5-epimerase expression is decreased in lung cancer cells}

Multiplex and quantitative RT-PCR analysis was used to study GLCE expression in lung cancer cells. Specific primers and TaqMan probes for the exon 2-3 boundary region of the GLCE gene (which appears to be affected in human tumours) were used (Grigorieva et al, 2008). D-Glucuronyl C5-epimerase expression was examined in U2020 (small-cell lung carcinoma), A716, A549, H647 and H157 (non-small-cell lung cancer) cells. Normal human lung cDNA (Clontech, San Diego, CA, USA) was used as a control for epimerase expression in normal lung tissues (Figures $1 \mathrm{~A}$ and $\mathrm{B}$ ).

According to the multiplex RT - PCR data, the GLCE expression levels in all of the tested cell lines was three to four times lower than in normal lung tissue (Figure 1A). The effect was quantified using TaqMan-based qRT-PCR, and GLCE expression was determined to be $1100-1200$ epimerase molecules per 1000 actin molecules in normal lung tissue and 100-200 epimerase molecules per 1000 actin molecules in the lung cancer cell lines (Figure 1B). These results clearly show that GLCE expression is significantly decreased (five to six times) in lung cancer cell lines compared with normal human lung tissues.

\section{D-Glucuronyl C5-epimerase re-expression suppresses U2020 cell proliferation but not viability in vitro}

To study the functional role of GLCE in lung cancer, the gene was ectopically expressed in U2020 cells. As a first step, full-length GLCE cDNA was cloned into the episomal vector $\mathrm{pETE} / \mathrm{Bsd}$ for the effective ectopic expression of GLCE in mammalian cells. The obtained DNA plasmid was designated as epi-pETE/Bsd. U2020 cells were transiently transfected with either epi-pETE/Bsd (epi-U2020) or an empty pETE/Bsd control vector (pETE-U2020) and subjected to colony formation assay and growth curve assay (Figure 2).

Colony formation was evaluated in monolayer cultures. The number of colonies formed by the epi-U2020 cells was similar to that formed by the control pETE-U2020 cells (Figures 2A and B). However, there was a significant difference in growth time between the epi-U2020 and the pETE-U2020 clones (6 weeks and 2 weeks

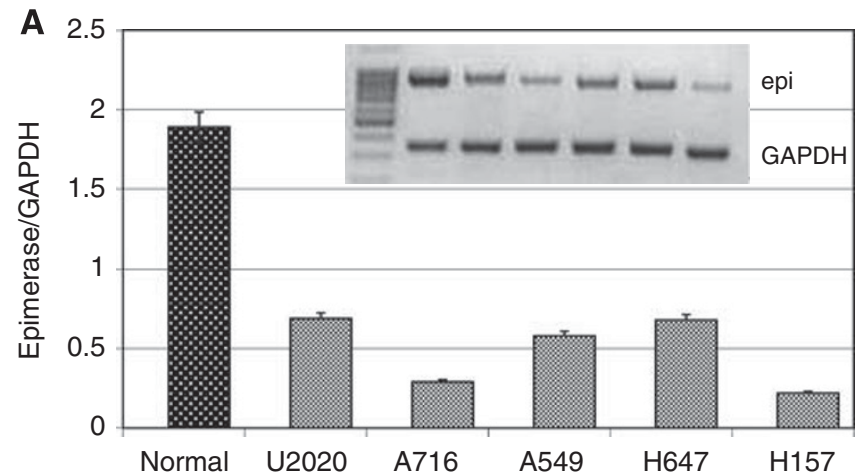

B

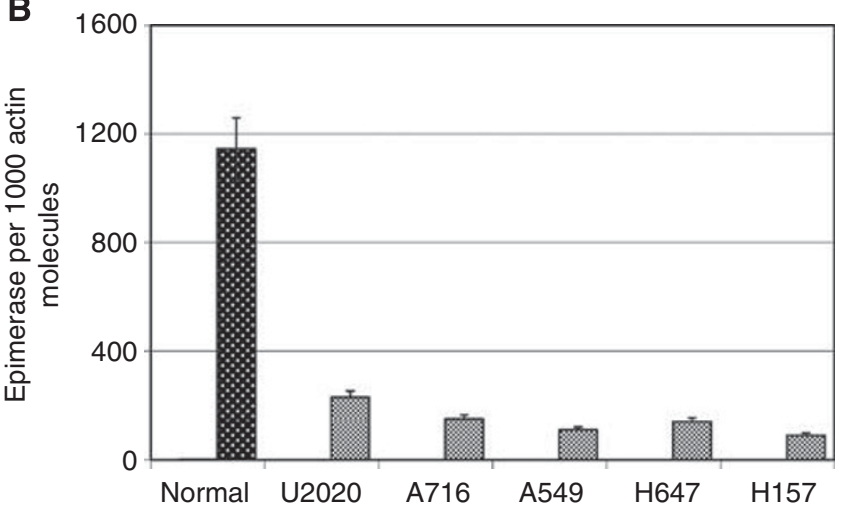

Figure I D-Glucuronyl C5-epimerase expression in human lung cancer cell lines. (A). Multiplex RT-PCR. Intensity of the amplified epimerase DNA fragments normalised to that of GAPDH. A representative gel is shown (inset). (B) Quantitative real-time RT-PCR. D-Glucuronyl C5-epimerase expression in normal human lung and lung cancer cell lines (U2020, A7 I6, A549, H647 and HI57). 
BJC $\cap$

A
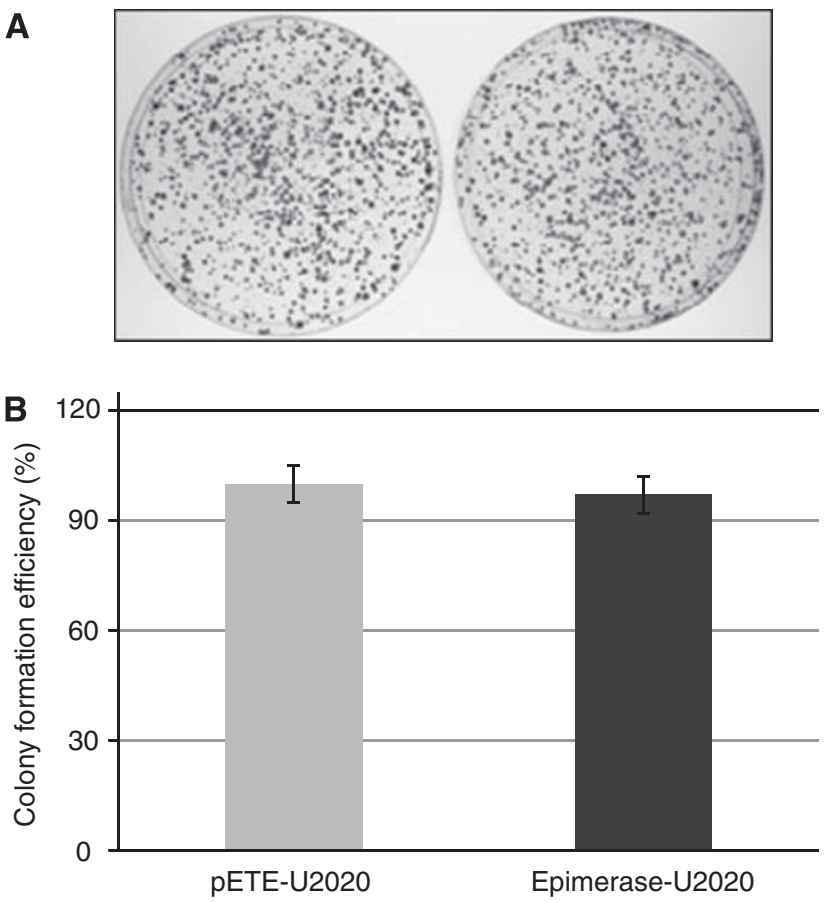

C
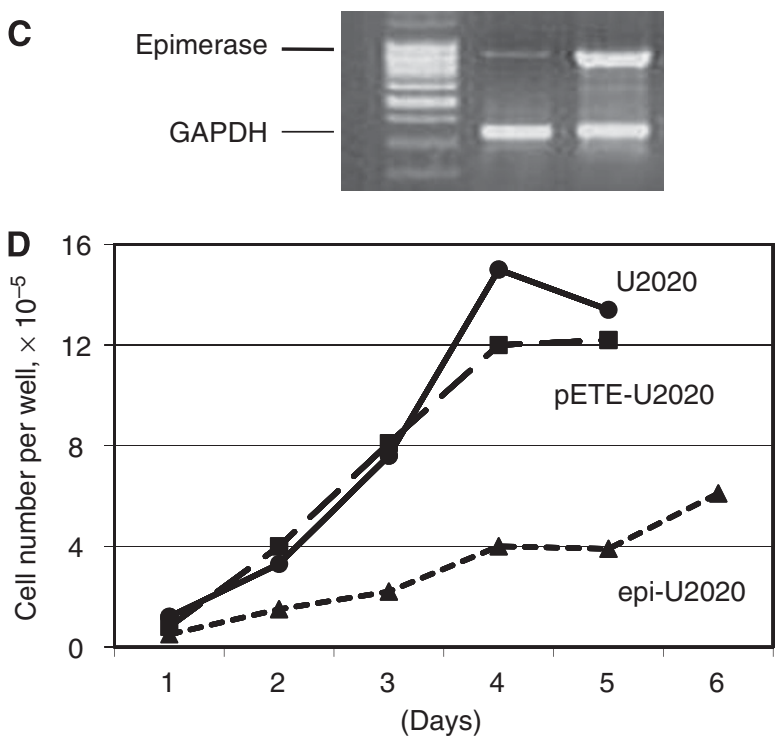

Figure 2 Effects of GLCE on the viability and proliferation of $U 2020$ cells in vitro. (A) The colony formation efficiency of epi-pETE- and pETEtransfected U2020 cells (transient transfection). Original plates stained with Giemsa are shown. (B) Quantitative analysis of colony numbers for the epi-U2020 and control pETE-U2020 plates. The number of colonies on the control pETE-U2020 plates was set as 100\%. The histogram shows the average colony numbers from triplicate experiments. Statistical analysis was performed using OriginPro 8.I (data represent the mean \pm s.d.). (C) Representative multiplex RT-PCR experiment showing the expression of GLCE. (D) Proliferation of epi-U2020 and pETE-U2020 cells (Growth curve assay).

between transfection and plate staining, respectively). To investigate the possible reasons for this difference, the proliferation of U2020 cells transiently transfected with either the epi-pETE/Bsd or the control pETE/Bsd plasmids was estimated using a growth curve assay (Figure 2C). The results showed that transfection with the epi-pETE/Bsd plasmid impaired U2020 cell proliferation compared with the control pETE-U2020 cells.
To further study the anti-proliferative effects of GLCE in U2020 cells, stable epi-U2020 and pETE-U2020 clones were selected. These clones were checked for GLCE expression using multiplex RT-PCR and the clones with the highest epimerase expression (clones 4, 7 and 8) were used for the subsequent experiments (Figures $3 \mathrm{~A}$ and $\mathrm{B}$ ).

The restored epimerase mRNA levels in the stable epi-U2020 clones were significantly higher than those in the control pETEU2020 clones, and were very similar to those in normal human lung tissue. The presence of GLCE protein in the epi-U2020 cells was verified by immunocytochemical staining using a custom anti-epimerase polyclonal antibody (Figure 3D). Interestingly, the stable epi-U2020 clones were morphologically different from the control pETE-U2020 clones (Figure 3C), whereas the control clones showed the same morphology as the original U2020 cells.

The proliferation rate of the stable epi-pETE and pETE-U2020 clones was determined using the CyQUANT NF Cell Proliferation Assay based on the cellular DNA content measured by binding of the fluorescent CyQUANT GR dye (Figure 3E). The stable epi-U2020 clones $(4,7$ and 8) proliferated five to six times more slowly than the control pETE-U2020 cells. These results showed that the selected stable epi-U2020 clones expressed GLCE at levels similar to that in normal human lung tissue, and that ectopic re-expression of GLCE in U2020 cells did not change their viability, but did affect their morphology and proliferation rate in vitro.

\section{D-Glucuronyl C5-epimerase inhibits U2020 tumour xenograft growth in vivo}

Epimerase-expressing U2020 cells (clones 4, 7 and 8) were inoculated into SCID mice to study the effects of GLCE on the growth of experimental lung tumours in vivo. U2020 cells stably transfected with an empty vector (pETE-U2020) were used as a control. Almost all the xenograft tumours grew more slowly than the control tumours (Figure 4A), or failed to grow at all. Over the course of 31-35 days, solid tumours were detected in 5 of 6 mice inoculated with pETE-U2020 cells (control tumours), but in only 5 of 10 mice inoculated with epi-U2020 cells. All the tumours (including the controls) were explanted and epimerase expression determined using multiplex RT - PCR and qRT-PCR (Figures 4B and C). The grown epi-U2020 xenograft tumours showed almost no epimerase expression, although the epi-U2020 cells used for the initial injections did show ectopic epimerase expression at levels similar to those in normal lung tissue (Figures $4 \mathrm{~B}$ and $\mathrm{C}$ ). qRT-PCR confirmed the multiplex RT-PCR data, estimating GLCE expression in the initial epi-U2020 cells to be 720-960 epimerase molecules per $1000 \mathrm{GAPDH}$ molecules, and almost no epimerase expression in the grown epi-U2020 xenograft tumours. Interestingly, slow-growing xenografts showed higher epimerase expression (similar to that of the initial epi-U2020 cells; Figures 4B and $\mathrm{C}$ ), indicating that epimerase inactivation in the growing experimental tumours may have been necessary for cancer progression.

These results show that GLCE expression in U2020 cells inhibits the growth of U2020 xenograft tumours in SCID mice and suggests that GLCE may be a potential, novel tumour-suppressor gene.

\section{D-Glucuronyl C5-epimerase affects lung cancer cell proliferation and tumour growth by inhibiting tumour angiogenesis and invasion/metastasis pathways}

To further elucidate the molecular mechanisms underlying the anti-proliferative effects of GLCE in small-cell lung cancer, we used the Cancer PathFinder RT Profiler PCR Array. This new highthroughput tool allows researchers to simultaneously profile the expression of a panel of 84 cancer-related genes involved in 6 different molecular pathways and combines the quantitative performance of SYBR Green-based RT-PCR with the multiple 
A

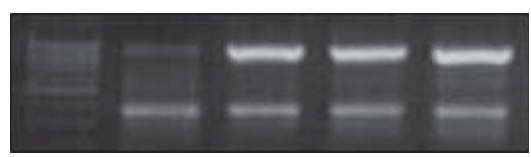

B

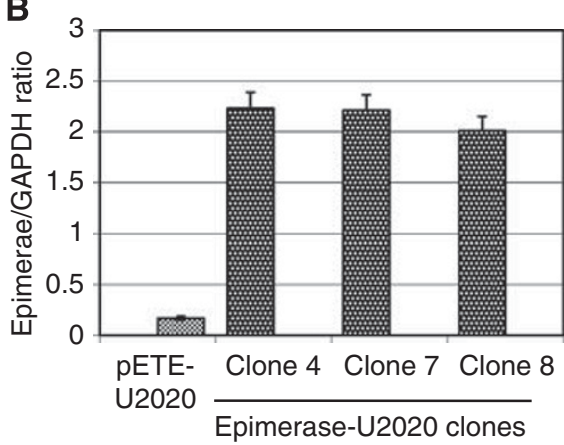

C

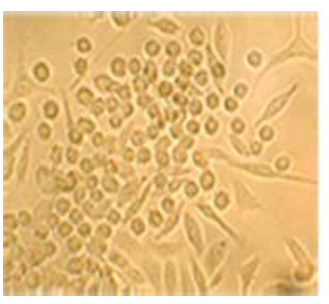

pETE-U2020

D

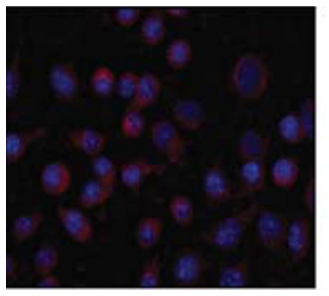

pETE-U2020

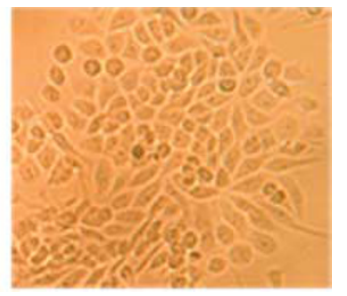

Epimerase-U2020

clone 7

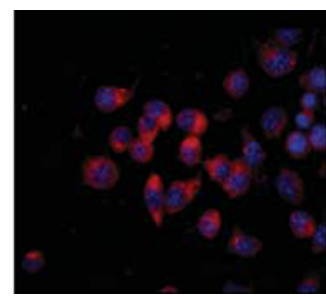

Epimerase-U2020 clone 7

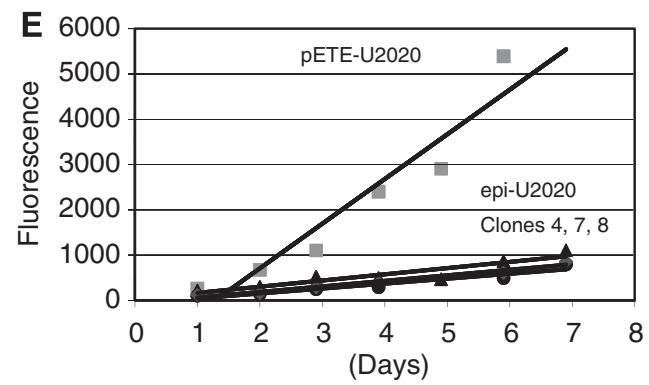

Figure 3 Ectopic expression of GLCE in U2020 small-cell lung cancer cells. (A) Representative multiplex RT-PCR gel showing GLCE expression in U2020 cells stably transfected with the pETE or epi-pETE plasmids (clones 4, 7 and 8). (B) GLCE expression levels normalised to that of GAPDH. The graph shows the mean expression levels from triplicate experiments ( \pm s.d.) (OriginPro 8.I). (C) Morphology of stably transfected pETE-U2020 cells and epi-U2020 cells (clone 7); magnification × 100. (D) Immunocytochemical analysis of GLCE expression in pETE-U2020 and epimerase-U2020 cells using a custom anti-epimerase polyclonal antibody. (E) Proliferation rates of the stable epi-U2020 and control pETE-U2020 clones (CyQUANT NF Cell proliferation assay).

gene-profiling capabilities of a microarray. The relative expression levels of each gene in the two samples are plotted against each other in a Scatter Plot (sample 1/control sample) and pair-wise comparison yields a fold change in the expression of the genes being studied.

We performed this analysis to look for genes whose expression was changed in epimerase-expressing U2020 cells compared with control pETE-U2020 cells. The results showed that the expression of 32 genes was either down- or upregulated by more than two-fold, while the expression of the remaining 52 genes was unaffected (Figure 5).

According to the Cancer PathFinder RT Profiler PCR Array, most of the affected genes are involved in apoptosis, angiogenesis and invasion/metastasis pathways.

Of the genes involved in apoptosis, TERT was downregulated 4.5-fold, APAF1 2.4-fold, HTATIP2 3.5-fold, and TNFa and the TNF receptors (TNFRSF10B, TNFRSF1A and TNFRSF25) 3-4-fold. Of those involved in angiogenesis, VEGF-A was downregulated 6.2-fold, TGFB1 3.1-fold, TNF 3.2-fold, FGFR2 3.2-fold, PDGF-A and PDGF-B 2.4-fold, and collagen XVIII 2.4-fold. Of those involved in invasion and metastasis, matrix metalloproteinase (MMP)2 was downregulated 8.3-fold, MTA1 4.3-fold, PLAU 4.3-fold, TIMP3 3-fold, S100A4 2.4-fold, SERPINE1 2.4-fold and TWIST1 3.7-fold.

The only other genes in U2020 cells affected by ectopic GLCE expression were cell cycle regulatory genes such as E2F-1
( -2.4 -fold) and p21 (-4.1-fold), the adhesion-related genes ITGA3 (-2.7-fold) and MCAM (-2.4-fold), and signal transductioninvolved genes SNCG (-6.2-fold), HER-2 (-2.4-fold), Jun (-3.2-fold) and AKT1 (-3.2-fold).

From these results, we hypothesise that changes in the expression levels of growth factors (VEGF-A, PDGF-A, PDGF-B, TGFB1, TNFa, IGF1) and their receptors (FGFR2, TGFB1R, TNFRSF10B, TNFRSF1A, TNFRSF25, FAS, HGFR/MET) may underpin the anti-proliferative effects of GLCE in U2020 cells in vitro (Figure 6).

Reduced growth factor expression, in combination with altered expression of key adhesion molecules (ITGA3, MCAM, collagen XVIII) may affect the adhesion, intercellular contacts and signalling in epi-U2020 cells, thereby creating a more physiologically 'normal' microenvironment and restoring contact inhibition of cell proliferation.

Taken together, these results show decreased GLCE expression in lung cancer cell lines compared with the normal lung tissue. Ectopic expression of GLCE in U2020 cells did not affect cell viability, but did suppress cell proliferation in vitro and the growth of experimental U2020 xenograft tumours in vivo. The in vitro and in vivo results from the Cancer PathFinder Array suggest that the anti-proliferative and anti-tumourigenic effects of GLCE in human small-cell lung cancer cells are mediated via downregulation of key growth factors and their receptors (particularly those involved in angiogenesis). 
A

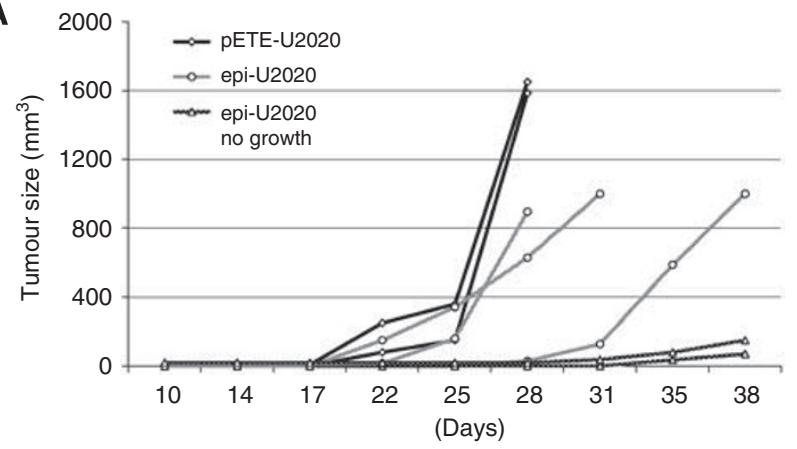

B
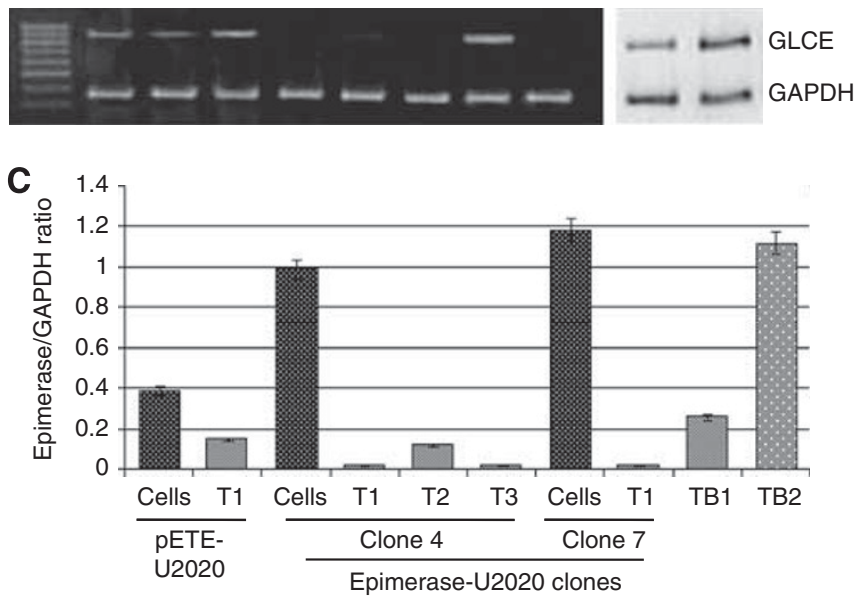

Figure 4 Effects of GLCE on tumour formation in SCID mice. (A) Tumour formation rate curve. Epi-U2020 (clones 4, 7 and 8) was used for inoculation. Three independent experiments were performed. (B and C) GLCE expression in mouse xenograft tumours. (B) Representative gel showing GLCE amplified by multiplex RT-PCR. (C) Intensity of the amplified epimerase DNA fragments normalised to that of GAPDH. Bars represent the mean \pm s.d. from triplicate experiments (OriginPro 8.I). The lane order on the gel corresponds to the bars on the histogram. Cells =epi-U2020 or pETE-U2020 cells; T=epi-U2020 or pETE-U2020 tumours; TB = slow-growing xenografts.

\section{DISCUSSION}

The results of the present study show that GLCE suppressed the proliferation of lung cancer cells in vitro, inhibited xenograft tumour formation in vivo and GLCE inactivation was necessary for the growth of experimental tumours in vivo. As shown previously (Li et al, 1999), this is a strong evidence for a tumour-suppressor function of $G L C E$ in lung cancer.

A similar effect was shown previously for other HS-synthesising enzymes, EXT1/EXT2, which are responsible for the polymerisation of the disaccharide-repeating regions within the growing HS chains (Lind et al, 1998). EXT1 expression is silenced in human cancer cells by epigenetic inactivation, and re-expression of EXT1 leads to the resumption of HS biosynthesis, reduced colony formation density and tumour growth in nude mice (Ropero et al, 2004). Unexpectedly, targeted deletion of EXT1 does not lead to a complete lack of HS synthesis. Indeed, two distinct cell lines defective in EXT1 expression produce a small, but significant, amount of HS chains (Okada et al, 2010), probably due to the contribution of other EXT gene family proteins (Kim et al, 2001).

D-Glucuronyl C5-epimerase is a single-copy gene working downstream of EXT1/EXT2 during HS biosynthesis. Epimerase knock-out mice show a lethal phenotype with lung and kidney defects (Li et al, 2003), suggesting the crucial importance of the gene in lung and kidney development. The results of the present study support an involvement of GLCE in lung carcinogenesis, and show that the gene is a second candidate tumour-suppressor gene related to the HS biosynthetic machinery along with EXT1.

According to the PathFinder Array results, three molecular pathways were mainly affected by ectopic GLCE expression in U2020 cells: apoptosis, angiogenesis and invasion/metastasis. With regard to apoptosis, the main changes involved decreased expression of TNF and TNF receptors (by 3-4-fold), which are involved in the early stages of apoptosis signalling/initiation. Taking into account the fact that expression of the main components of the intracellular apoptotic machinery (BCL2, $\mathrm{BAD}, \mathrm{BAX}, \mathrm{CASP} 8, \mathrm{GZMA})$ remained unchanged and the viability of epi-U2020 cells was not affected by ectopic expression of epimerase, we suppose that the TNF/TNF receptors have a nonapoptotic function in these cells. It may be that, in this case, the TNF/TNF receptors functioned mainly in angiogenesis-related events, and that the anti-mitotic effects of GLCE in U2020 cancer cells were not directly related to apoptosis.

Regarding angiogenesis, the main changes involved decreased expression of the pro-angiogenic growth factors, VEGF-A, PDGF-A, PDGF-B, FGFR2, TGFB1 and TNF. Most of these factors are upregulated in cancers. In particular, high expression of VEGF has been observed in many cancers and is associated with a poor prognosis (Azam et al, 2010). For example, VEGF expression increases as bronchial carcinoma progresses (Merrick et al, 2005) and is significantly increased in lung carcinomas (Brueckl et al, 2008). Over-expression of PDGF is linked to different types of malignancy (Appelmann et al, 2010). FGFR2 expression is significantly increased in cancer tissues (Marzioni et al, 2009) and aberrant activation of FGFR2 signalling induces the proliferation and survival of tumour cells (Katoh and Katoh, 2009). TGF- $\beta$, a tumour suppressor in normal or dysplastic cells, becomes a tumour promoter in advanced cancers (Nagaraj and Datta, 2010). Thus, the angiogenic growth factor 'cocktail' in cancers may be biased towards VEGF and assorted other factors like PDGF, FGF, EGF-like factors, angiopoietins (ANG1 and ANG2) or cytokines such as IL8 (Schulz, 2005).

Ectopic expression of GLCE significantly decreased VEGF-A expression (by 6.2-fold), as well as that of PDGF-A, PDGF-B and FGFR-2, while the expression of other pro-angiogenic genes (ANG1, ANG2 and IL8) remained unaffected. It is possible that GLCE re-balances the growth factor 'cocktail' in favour of tumour angiogenesis inhibition. If this is the case, then GLCE may be a novel, natural regulator of angiogenesis and a possible target for anticancer therapy.

The mechanism by which increased GLCE expression affects growth factor expression and angiogenesis may act via changes in the structure of cell surface and ECM proteoglycans. Many HSPGs are involved in angiogenesis, including syndecans (Alexopoulou et al, 2007), perlecan (Bix; Iozzo, 2005; Whitelock et al, 2008) and glypican-1 (Aikawa et al, 2008). For example, perlecan modulates VEGF - VEGFR2 signalling events during developmental angiogenesis (Zoeller et al, 2009); syndecans are necessary for FGFR tyrosine kinase receptor function - syndecans that bind both bFGF and FGFRs may act as stimulators of FGF signalling, whereas syndecans that only bind bFGF may act as inhibitors (Zhang et al, 2009). Thus, the fine structure of syndecans (particularly their HS chains) may provide the microenvironmental 'context' that determines the pro-oncogenic or anti-oncogenic function of FGFRs.

Several other studies show significant direct effects of HSPGs on gene expression. For example, over-expression of syndecan-1 influences the expression profiles of other syndecans (Zong et al, 2010) and results in increased nuclear accumulation of FGF-2 (Zong et al, 2009). This suggests the existence of a general mechanism for the regulation of gene expression by HSPGs.

Downregulation of GLCE expression/function in cancer cells may affect the structure of all the HSs and alter the cell 

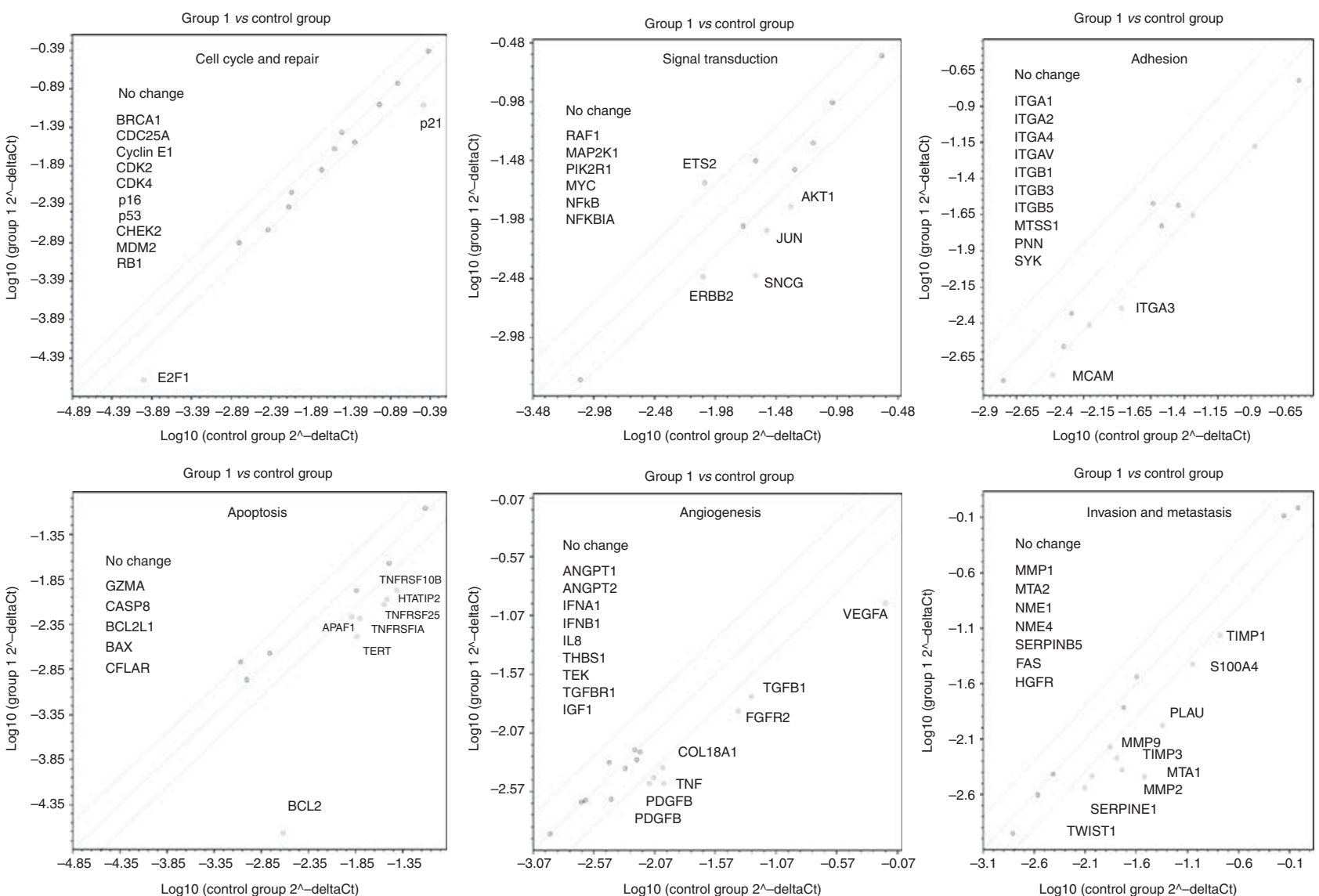

Figure 5 Cancer PathFinder RT2 Profiler PCR Array analysis of epimerase-expressing U2020 cells. The relative expression levels for each gene in epimerase-expressing U2020 cells (Group I) are plotted against the same gene from the control pETE-U2020 cells (Control Group). The middle line shows the similar expression in both groups with two-fold change boundaries. Genes upregulated greater than two-fold in epi-U2020 cells lie above the middle line and the downregulated genes lie below the line.

microenvironment, such that 'normal' angiogenesis switches to a malignant process.

The most significant changes to the pathways involved in invasion and metastasis were related to the downregulation of MMP2, uPA (PLAU) and the metastasis-associated genes, MTA1 and $S 1004 A$. All are associated with a highly metastatic cell phenotype. Matrix metalloproteinases are required for peritumour tissue degradation and metastasis (Overall and López-Otín, 2002), and their expression is generally restricted to aggressive lungmetastatic populations of cancer cells (Minn et al, 2005). uPA and its inhibitor, PAI-I, have a key role in tumour invasion and metastasis (Harbeck et al, 2004), and their expression levels are increased in angiogenic non-small-cell lung tumours (Offersen et al, 2007). MTA1 is a member of a newly discovered family of cancer progression-related genes that are over-expressed in a wide range of human cancers (Toh and Nicolson, 2009), and expression of the metastasis-associated gene, S100A4, is strongly correlated with an aggressive metastatic phenotype (Helfman et al, 2005). Ectopic expression of GLCE in U2020 cells decreased the expression of MMP2, uPA and the metastasis-associated genes, MTA1 and S1004A. This suggests that GLCE may also have potential anti-metastatic effects in vivo. Overall, the results of the present study are consistent with those of previous work investigating the anti-proliferative effects of GLCE over-expression in breast cancer cells in vitro (Prudnikova et al, 2010). In both cases, angiogenesis and invasion are thought to be the molecular mechanisms affected by GLCE.

These in vitro results are supported by those from the in vivo experiments comparing the relative expression levels of the same

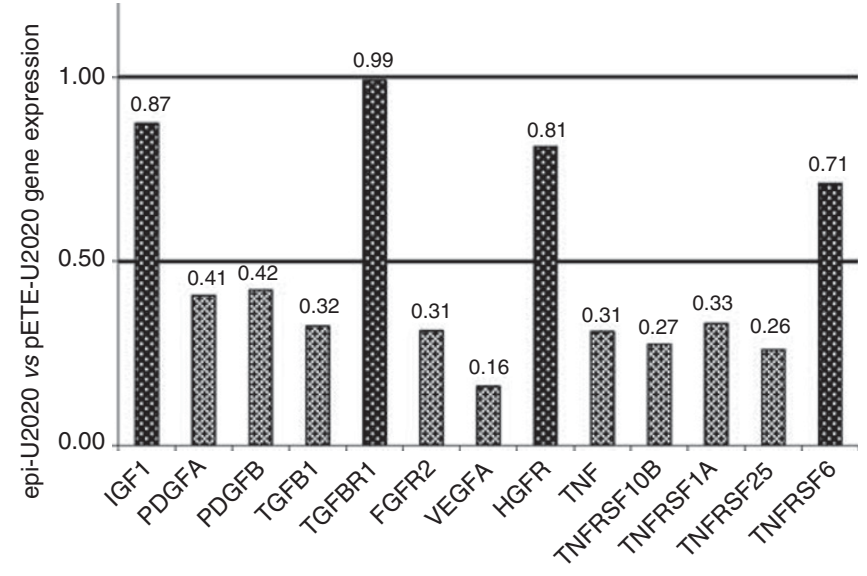

Figure 6 The relative expression of growth factors and growth factor receptors in epimerase-expressing U2020 cells versus control pETE-U2020 cells. I = Equal expression levels in epimerase-expressing and control cells; $0.50=$ two-fold decrease in expression.

genes in slow-growing xenografts (in which epimerase was expressed) and grown xenografts (epimerase inactivated) (Mostovich et al, 2010). Upregulation of IGF1, HTATIP2, BCL2, TNF, RB1, TNFRSF6, IFNA1, IFNB1, ITGA1, ITGA3, SNCG, NME1, BCL2L1, TGFB1 and FGFR2 in slow-growing xenografts coupled with the downregulation of PDGF-A, CFLAR, ITGB5, TNFRSF10B, MMP9, 
ANGPT1, SERPINE1, THBS1, ITGB3, p21, MET and ETS supports the hypothesis that angiogenesis- and invasion-related genes may be the downstream targets of epimerase in U2020 cells. The fact that the expression of other molecules (for e.g., pro-apoptotic or cell cycleregulating genes) was also affected in the in vivo experiments may reflect the complex nature of tumour-host interactions during tumour development, where the surrounding cells and ECM contribute to the overall expression profile.

Taken together, the results of the present study strongly suggest that GLCE is a novel regulator of cancer cell proliferation and tumour growth, and may be a promising target for lung cancer diagnosis and treatment.

\section{ACKNOWLEDGEMENTS}

We thank Professor M Lerman for his kind attention and critical reading of the manuscript. The work was supported by the research grant from Russian Foundation for Basic Research (RFBR 09-04-01599a); by a UICC International Cancer Technology Transfer Fellowship (EVG, ICR/08/086; TYP, ICR/09/069); Karolinska Institute (2008Fobi1152); EVG was recipient of fellowship from the Concern Foundation in Los Angeles and the Cancer Research Institute in New York; ERZ was supported by research grants from the Swedish Cancer Society, the Swedish Research Council and the Swedish Institute.

\section{REFERENCES}

Aikawa T, Whipple CA, Lopez ME, Gunn J, Young A, Lander AD, Korc M (2008) Glypican-1 modulates the angiogenic and metastatic potential of human and mouse cancer cells. J Clin Invest 118: 89-99

Alexopoulou AN, Multhaupt HA, Couchman JR (2007) Syndecans in wound healing, inflammation and vascular biology. Int J Biochem Cell Biol 39: $505-528$

Appelmann I, Liersch R, Kessler T, Mesters RM, Berdel WE (2010) Angiogenesis inhibition in cancer therapy: platelet-derived growth factor (PDGF) and vascular endothelial growth factor (VEGF) and their receptors: biological functions and role in malignancy. Recent Results Cancer Res 180: $51-81$

Azam F, Mehta S, Harris AL (2010) Mechanisms of resistance to antiangiogenesis therapy. Eur J Cancer 46(8): 1323-1332

Blackhall FH, Merry CL, Davies EJ, Jayson GC (2001) Heparan sulfate proteoglycans and cancer. Br J Cancer 85: 1094-1098

Brueckl WM, Schoeberl A, Wirtz RM, Murray S, Hahn EG, Wiest GH (2008) Increased vascular-endothelial growth factor (VEGF) tumor expression and response to epidermal growth factor receptor (EGF-R) inhibitor erlotinib in non-small cell lung cancer (NSCLC). J Thorac Oncol 3: $314-316$

Catlow KR, Deakin JA, Wei Z, Delehedde M, Fernig DG, Gherardi E, Gallagher JT, Pavão MS, Lyon M (2008) Interactions of hepatocyte growth factor/scatter factor with various glycosaminoglycans reveal an important interplay between the presence of iduronate and sulfate density. J Biol Chem 283: 5235-5248

Crawford BE, Olson SK, Esko JD, Pinhal MA (2001) Cloning, Golgi localization, and enzyme activity of the full-length heparin/heparan sulfate-glucuronic acid C5-epimerase. J Biol Chem 276: $21538-21543$

Eshchenko TY, Rykova VI, Chernakov AE, Sidorov SV, Grigorieva EV (2007) Expression of different proteoglycans in human breast tumors. Biochemistry (Mosc) 72: 1016-1020

Filmus J, Capurro M, Rast J (2008) Glipicans. Genome Biol 9: 224

Grigorieva E, Eshchenko T, Rykova VI, Chernakov A, Zabarovsky E, Sidorov SV (2008) Decreased expression of human D-glucuronyl C5-epimerase in breast cancer. Int J Cancer 122: $1172-1176$

Hann CL, Rudin CM (2007) Fast, hungry and unstable: finding the Achilles' heel of small-cell lung cancer. Trends Mol Med 13: 150-157

Harbeck N, Kates RE, Gauger K, Willems A, Kiechle M, Magdolen V, Schmitt M (2004) Urokinase-type plasminogen activator (uPA) and its inhibitor PAI-I: novel tumor-derived factors with a high prognostic and predictive impact in breast cancer. Thromb Haemost 91: 450-456

Helfman DM, Kim EJ, Lukanidin E, Grigorian M (2005) The metastasis associated protein S100A4: role in tumour progression and metastasis. Br J Cancer 92: $1955-1958$

Iozzo RV (2005) Basement membrane proteoglycans: from cellar to ceiling. Nat Rev Mol Cell Biol 6: 646-656

Iozzo RV, Zoeller JJ, Nyström A (2009) Basement membrane proteoglycans: modulators Par Excellence of cancer growth and angiogenesis. Mol Cells 27: $503-513$

Jia J, Maccarana M, Zhang X, Bespalov M, Lindahl U, Li JP (2009) Lack of L-iduronic acid in heparan sulfate affects interaction with growth factors and cell signaling. J Biol Chem 284: $15942-15950$

Katoh Y, Katoh M (2009) FGFR2-related pathogenesis and FGFR2-targeted therapeutics. Int J Mol Med 23: $307-311$

Kim BT, Kitagawa H, Tamura J, Saito T, Kusche-Gullberg M, Lindahl U, Sugahara K (2001) Human tumor suppressor EXT gene family members
EXTL1 and EXTL3 encode alpha 1,4- N-acetylglucosaminyltransferases that likely are involved in heparan sulfate/ heparin biosynthesis. Proc Natl Acad Sci USA 98(13): 7176-7181

Kim H, Xu GL, Borczuk AC, Busch S, Filmus J, Capurro M, Brody JS, Lange J, D'Armiento JM, Rothman PB, Powell CA (2003) The heparan sulfate proteoglycan GPC3 is a potential lung tumor suppressor. Am J Respir Cell Mol Biol 29: 694-701

Lander AD, Selleck SB (2000) The elusive functions of proteoglycans: in vivo veritas. J Cell Biol 148: $227-232$

Li J, Protopopov AI, Gizatullin RZ, Kiss C, Kashuba VI, Winberg G, Klein G, Zabarovsky ER (1999) Identification of new tumor suppressor genes based on in vivo functional inactivation of a candidate gene. FEBS Lett 451(3): $289-294$

Li JP, Gong F, El Darwish K, Jalkanen M, Lindahl U (2001) Characterization of the D-glucuronyl C5-epimerase involved in the biosynthesis of heparin and heparan sulfate. J Biol Chem 276: 20069-20077

Li JP, Gong F, Hagner-McWhirter A, Forsberg E, Abrink M, Kisilevsky R, Zhang X, Lindahl U (2003) Targeted disruption of a murine glucuronyl C5-epimerase gene results in heparan sulfate lacking L-iduronic acid and in neonatal lethality. J Biol Chem 278: 28363-28366

Li JP, Hagner-McWhirter A, Kjellén L, Palgi J, Jalkanen M, Lindahl U (1997) Biosynthesis of heparin/heparan sulfate. cDNA cloning and expression of D-glucuronyl C5-epimerase from bovine lung. J Biol Chem 272: $28158-28163$

Lind T, Tufaro F, McCormick C, Lindahl U, Lidholt K (1998) The putative tumor suppressors EXT1 and EXT2 are glycosyltransferases required for the biosynthesis of heparan sulfate. J Biol Chem 273(41): 26265-26268

Marzioni D, Lorenzi T, Mazzucchelli R, Capparuccia L, Morroni M, Fiorini R, Bracalenti C, Catalano A, David G, Castellucci M, Muzzonigro G, Montironi R (2009) Expression of basic fibroblast growth factor, its receptors and syndecans in bladder cancer. Int J Immunopathol Pharmacol 22: $627-638$

Merrick DT, Haney J, Petrunich S, Sugita M, Miller YE, Keith RL, Kennedy TC, Franklin WA (2005) Overexpression of vascular endothelial growth factor and its receptors in bronchial dypslasia demonstrated by quantitative RT-PCR analysis. Lung Cancer 48: 31 - 45

Minn AJ, Gupta GP, Siegel PM, Bos PD, Shu W, Giri DD, Viale A, Olshen AB, Gerald WL, Massagué J (2005) Genes that mediate breast cancer metastasis to lung. Nature 436: 518-524

Mostovich LA, Prudnikova TY, Domanitskaya NV, Verzhbitskaya NE, Kharchenko OV, Nepomnyaschikh GI, Aidagulova SV, Rykova VI, Grigoryeva EV (2010) in vivo. Siber J Oncol 2: 24-29

Nackaerts K, Verbeken E, Deneffe G, Vanderschueren B, Demedts M, David G (1997) Heparan sulfate proteoglycan expression in human lungcancer cells. Int J Cancer 74: $335-345$

Nadanaka S, Kitagawa H (2008) Heparan sulphate biosynthesis and disease. J Biochem 144: 7 - 14

Nagaraj NS, Datta PK (2010) Targeting the transforming growth factor-beta signaling pathway in human cancer. Expert Opin Investig Drugs 19: 77-91

Offersen BV, Pfeiffer P, Andreasen P, Overgaard J (2007) Urokinase plasminogen activator and plasminogen activator inhibitor type- 1 in nonsmall-cell lung cancer: relation to prognosis and angiogenesis. Lung Cancer 56: $43-50$

Okada M, Nadanaka S, Shoji N, Tamura JI, Kitagawa H (2010) Biosynthesis of heparan sulfate in EXT1-deficient cells. Biochem J 428: 463-471 
Ori A, Wilkinson MC, Fernig DG (2008) The heparanome and regulation of cell function: structures, functions and challenges. Front Biosci 13: $4309-4338$

Overall CM, López-Otín C (2002) Strategies for MMP inhibition in cancer: innovations for the post-trial era. Nat Rev Cancer 2: 657-672

Pastorino U (2010) Lung cancer screening. Br J Cancer 102: 1681 - 1686

Protopopov AI, Li J, Winberg G, Gizatullin RZ, Kashuba VI, Klein G, Zabarovsky ER (2002) Human cell lines engineered for tetracyclineregulated expression of tumor suppressor candidate genes from a frequently affected chromosomal region, 3p21. J Gene Med 4: 397-406

Prudnikova TY, Mostovich LA, Domanitskaya NV, Pavlova TV, Kashuba VI, Zabarovsky ER, Grigorieva EV (2010) Antiproliferative effect of D-glucuronyl C5-epimerase in human breast cancer cells. Cancer Cell Int 10: 27

Reijmers RM, Groen RW, Rozemuller H, Kuil A, de Haan-Kramer A, Csikós T, Martens AC, Spaargaren M, Pals ST (2010) Targeting EXT1 reveals a crucial role for heparan sulfate in the growth of multiple myeloma. Blood 115: 601-604

Ropero S, Setien F, Espada J, Fraga MF, Herranz M, Asp J, Benassi MS, Franchi A, Patiño A, Ward LS, Bovee J, Cigudosa JC, Wim W, Esteller M (2004) Epigenetic loss of the familial tumor-suppressor gene exostosin-1 (EXT1) disrupts heparan sulfate synthesis in cancer cells. Hum Mol Genet 13: $2753-2765$

Rosti G, Bevilacqua G, Bidoli P, Portalone L, Santo A, Genestreti G (2006) Small cell lung cancer. Ann Oncology 17(Suppl 2): ii5-ii10

Schulz WA (2005) Molecular Biology of Human Cancers, p 208. AA Dordrecht: The Netherlands
Toh Y, Nicolson GL (2009) The role of the MTA family and their encoded proteins in human cancers: molecular functions and clinical implications. Clin Exp Metastasis 26: 215-227

Whitelock JM, Melrose J, Iozzo RV (2008) Diverse cell signaling events modulated by perlecan. Biochemistry 47: 11174-11183

Zhang C, Fu L, Fu J, Hu L, Yang H, Rong TH, Li Y, Liu H, Fu SB, Zeng YX, Guan XY (2009) Fibroblast growth factor receptor 2-positive fibroblasts provide a suitable microenvironment for tumor development and progression in esophageal carcinoma. Clin Cancer Res 15: 4017-4027

Zoeller JJ, Whitelock JM, Iozzo RV (2009) Perlecan regulates developmental angiogenesis by modulating the VEGF-VEGFR2 axis. Matrix Biol 28: $284-291$

Zong F, Fthenou E, Castro J, acute;terfia B, Kovalszky I, lák L, Tzanakakis G, Dobra K (2010) Effect of syndecan-1 overexpression on mesenchymal tumour cell proliferation with focus on different functional domains. Cell Prolif 43: 29-40

Zong F, Fthenou E, Wolmer N, Hollo'si P, Kovalszky I, Szilák L, Mogler C, Nilsonne G, Tzanakakis G, Dobra K (2009) Syndecan-1 and FGF-2, but not FGF receptor-1, share a common transport route and co-localize with heparanase in the nuclei of mesenchymal tumor cells. PLoS One 4(10): e7346

(c) (1) This work is licensed under the Creative Commons Attribution-NonCommercial-Share Alike 3.0 Unported License. To view a copy of this license, visit http://creativecommons. org/licenses/by-nc-sa/3.0/ 